\title{
Evaluation of Chitin and Chitosan as a Sorbent for the Preconcentration of Phenol and Chlorophenols in Water
}

\author{
Jae-Seong RheE*广, Min-Woo Jung** and Ki-Jung PAEnG** \\ * Water Environmental Research Center, KIST, P.O.Box 131, Cheongryangri, Seoul 130-650, Korea \\ **Department of Chemistry, Yonsei University, Wonju 220-710, Korea
}

\begin{abstract}
The evaluation of the applicability of chitin and chitosan as a sorbent material to solid phase extraction of phenol and chlorophenols was made by using high-performance liquid chromatography (HPLC). For the measurement of the adsorptive capabilities, adsorption isotherms as well as breakthrough curves were obtained by means of batch experiments or column experiments respectively. The increasing tendency of the amount of adsorption with the number of chlorine atom in phenol molecules was observed for both chitin and chitosan. The driving force of the interaction could be attributed to hydrogen bonding among electro-negative atoms in chitin, chitosan or chlorophenols.
\end{abstract}

Keywords Chitin, chitosan, sorbent, solid phase extraction, phenol, chlorophenol, adsorptive capability

Chitin, poly- $\beta$ - $(1 \rightarrow 4)-N$-acetyl-D-glucosamine, the main component of shells of Crustacea such as crabs, shrimps and various insects is the most widely occurring natural biopolymer along with cellulose. Chitin is clustered with calcium carbonate and proteins whereas chitosan is prepared from chitin by deacetylating its acetamide groups with a strong alkaline solution. ${ }^{1,2}$ Though chitosan is found naturally in the cell walls of certain fungi, these sources are not yet utilized for industrial use. ${ }^{3}$ At the present, chitosan is obtained predominantly through alkaline deacetylation of chitin.

Since chitin and chitosan have various bioactivities and functionalities, they attract much attention and are studied in various fields of application. ${ }^{4-8}$ While chitin is insoluble in most solvents, chitosan is readily soluble in acidic solutions and easily available for industrial purposes. ${ }^{9}$ Many applications are due to the secondary amino groups of chitosan which show polycationic, chelating and film-forming properties along with high solubility in dilute acetic acid. ${ }^{10}$

For the chromatographic determination of low concentrations of environmental organic pollutants, especially in complex matrices, the preconcentration of samples is necessary. Considerable attention is being paid to solid phase extraction (SPE) as an alternative over the laborious and time-consuming liquid-liquid extraction (LLE). Various types of sorbents, such as octadecyl-bonded silica $\left(\mathrm{C}_{18}\right)^{11}$, porous polymeric resins ${ }^{12}$, carbon-based sorbents ${ }^{13}$, ion-exchange resins ${ }^{14}$ are being used for that purpose.

At the present study, the applicability of chitin and chitosan as a sorbent material in solid phase extraction of environmental organic contaminants like chlorophe-

† To whom correspondence should be addressed. nols was evaluated by using a high-performance liquid chromatograph equipped with a diode-array detector (DAD).

\section{Experimental}

\section{Chemicals and reagents}

Phenol (Ph), 2-chlorophenol (2-CP), 4-chlorophenol (4-CP), 2,4-dichloro-phenol (2,4-DCP), 2,4,6trichlorophenol (2,4,6-TCP), pentachlorophenol (PCP) were obtained from Fluka (Ronkonkoma, NY, USA). Stock solutions of $2000 \mathrm{mg} / \mathrm{l}$ of each compound were prepared with methanol and stored in a refrigerator. Working solutions were prepared by diluting the stock solutions with methanol just prior to use. HPLC-grade acetonitrile was obtained from J. T. Baker (Phillipsburg, NJ, USA). Distilled water was further purified with an ultrapure cartridge system (Barnstead/ Thermolyne, USA). All other reagents were of analytical grade. For the preparation of the mobile phase, acetonitrile or water were filtered through a $0.2 \mu \mathrm{m}$ nylon filter (Whatman, Maldstone, England) or $0.45 \mu \mathrm{m}$ cellulose nitrate filter (Whatman) respectively.

\section{Apparatus}

Chromatographic experiments were performed using a Hewlett-Packard (Waldbronn, Germany) 1050 Series ternary gradient liquid chromatograph with a diodearray detector. The system control and data analysis were executed by a HPLC3D Chemstation (DOS series). Separation was carried out using a Mightysil RP-18 GP C18 column $(250 \times 4.6$ mm i.d., $5 \mu \mathrm{m})$ from Kanto Chemical Co., Inc. (Tokyo, Japan). A Rheodyne Model 7125 injector having a $20 \mu l$ loop was used. 


\section{Chromatographic conditions and detection}

The chromatographic separations were carried out by a gradient of acetonitrile and $10^{-3} \mathrm{M}$ phosphoric acid mixing from 38:62 to $95: 5$ within $20 \mathrm{~min}$. The mobile phase was degassed by helium $(99.99 \%)$ purging during the gradient elution. In order to determine the optimum wavelengths for detection, UV spectra were recorded from 190 to $500 \mathrm{~nm}$ for each phenol. The detection wavelength was set at $225 \mathrm{~nm}$ in case of monitoring all phenols simultaneously.

\section{Sorbents}

Chitin and chitosan were obtained from Sigma (St. Louis, MO, USA). They were ground and sieved to 100 - 150 mesh, followed by purifying with a Soxhlet extraction and sonication in the media of organic solvents sequentially. The structures of chitin and chitosan are shown in Fig. 1.

\section{Batch type determination}

For the determination of the relationship between the amount of sorbent and the amount adsorbed, 25, 50, $100 \mathrm{mg}$ of sorbents with aliquots of standard solution in water ( $\mathrm{pH} 3$ adjusted with hydrochloric acid) were mixed in a glass vial followed by shaking for $30 \mathrm{~min}$ with a Fisher Vortex-Genie 2 Mixer (Fisher Scientific, Pittsburgh, PA, USA).

In order to obtain the adsorption isotherms, $100 \mathrm{mg}$ of each sorbent, standard solution and water $(\mathrm{pH} 3$ with $\mathrm{HCl}$ ) were mixed in a glass vial to give the desired concentration of each compound. It was also shaken for 30 min in order to reach equilibrium. Twenty microliters of aliquot, after filtering through a $0.45 \mu \mathrm{m}$ PVDF syringe filter (Whatman), were injected to the HPLC.

\section{Experiment with column}

For the measurements of breakthrough volume, a stainless-steel column $(20 \times 2 \mathrm{~mm}$ i.d. $)$ was introduced. The sorbent was slurry-packed with methanol under

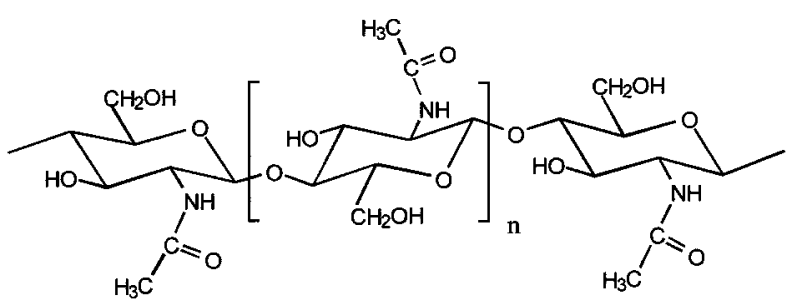

Chitin

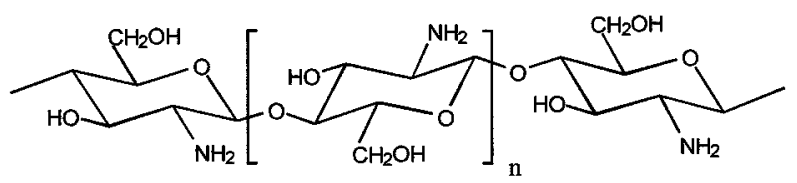

Chitosan

Fig. 1 Chemical structure of chitin and chitosan. vacuum and the top and bottom of each sorbent bed were fitted with stainless-steel frits (10 $\mu \mathrm{m}$ pore size).

Before measurements, sorbents were flushed with water ( $\mathrm{pH} 3$ with $\mathrm{HCl}$ ) in order to remove the remaining methanol until the baseline stability was obtained. To obtain breakthrough curves, a standard solution of $0.1 \mathrm{mg} / \mathrm{l}$ of phenols dissolved in water at $\mathrm{pH} 3$ (with $\mathrm{HCl}$ ) was pumped through the column and directly introduced to the detector at a flow rate of $2.5 \mathrm{ml} / \mathrm{min}$.

\section{Results and Discussion}

\section{Purification of chitin and chitosan}

The impurities in the sorbent should be removed until satisfactory background was obtained because the presence of trace impurities has been shown to exert a vital effect on the analysis. Each sorbent was treated with Soxhlet extractor for $6 \mathrm{~h}$ following sonication for 30 min in methanol and acetonitrile.

The extracts of each solvent were concentrated with a rotary evaporator to a volume of $1 \mathrm{ml}$ for examination of purity by HPLC under the same condition as for analytes with gradient elution. The purification step was repeated until the blank chromatogram reached a clear response beyond the range of interferences.

\section{Breakthrough volumes}

Breakthrough measurements were carried out only for chitin because chitosan swelled in aqueous phase. The breakthrough curves for selected phenols on chitin are shown in Fig. 2.

If the breakthrough volume is taken as the volume at which the signal reaches $5 \%$ of the total signal, the breakthrough volumes obtained for phenol, 2chlorophenol, 2,4,6-trichlorophenol, pentachlorophenol were measured as 2.6, 2.8, 3.9 and $11.3 \mathrm{ml}$ respectively. The breakthrough volume was found to be dependent

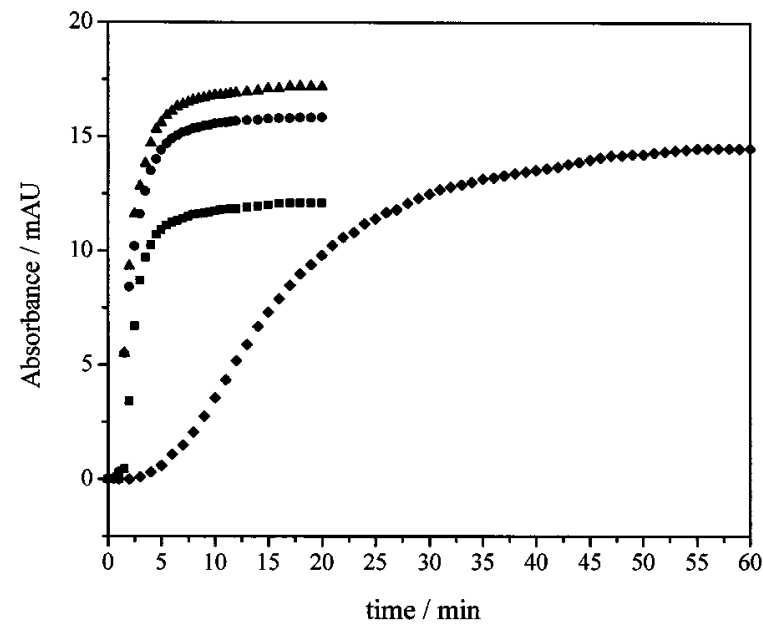

Fig. 2 Breakthrough curves of $\mathrm{Ph}(\bullet)$, 2-CP ( $)$, 2,4,6-TCP ( $)$ and PCP ( ) recorded with a $20 \times 2 \mathrm{~mm}$ i.d. precolumn packed with chitin. 


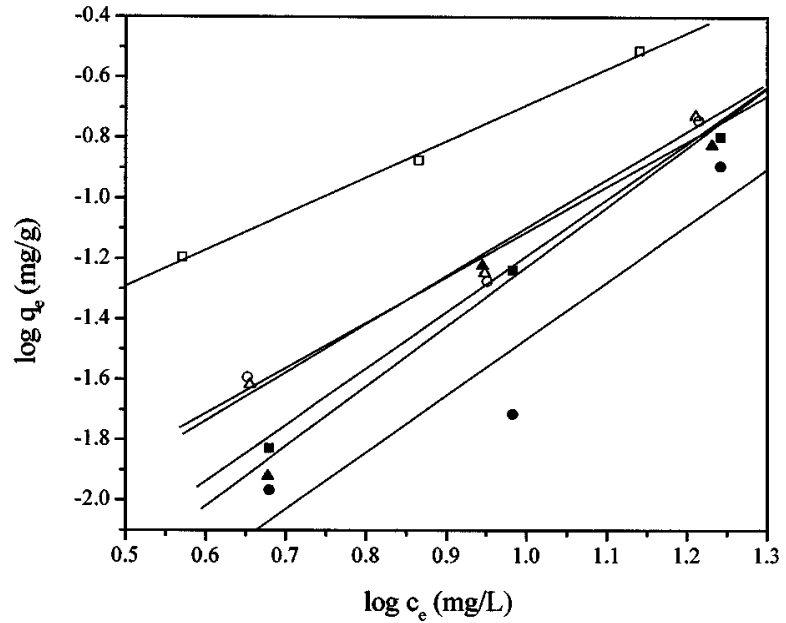

Fig. 3 Freundlich plots of individual adsorption isotherms for

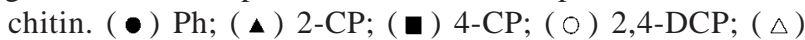
2,4,6-TCP; ( $\square$ ) PCP.

on the number of substituents in phenol molecules. As the number of substituents was increased, the breakthrough volume was also increased. It can be predicted that the main interaction in adsorption between chlorophenols and chitin is attributed to hydrogen bonding between chlorine, oxygen atoms in phenol molecules and hydrogen atoms in chitin showing more affinity as the number of chlorine atoms substituted increased.

\section{Adsorption isotherms}

In general, the adsorption isotherm defines the distribution of a solute between the ligand and solid phases after the adsorptive reaction has reached an equilibrium condition. Two of the most commonly used isotherms are the Langmuir and the Freundlich equations. Since the Langmuir equation gave a relatively poor fit, the applicability of Freundlich equation was examined.

The Freundlich equation represents an empirical relationship of the amount of adsorbate in the adsorbed phase to that in solution:

$$
q_{\mathrm{e}}=K_{\mathrm{F}} C_{\mathrm{e}}^{1 / n}
$$

where $q_{\mathrm{e}}$ is the amount adsorbed per unit mass of

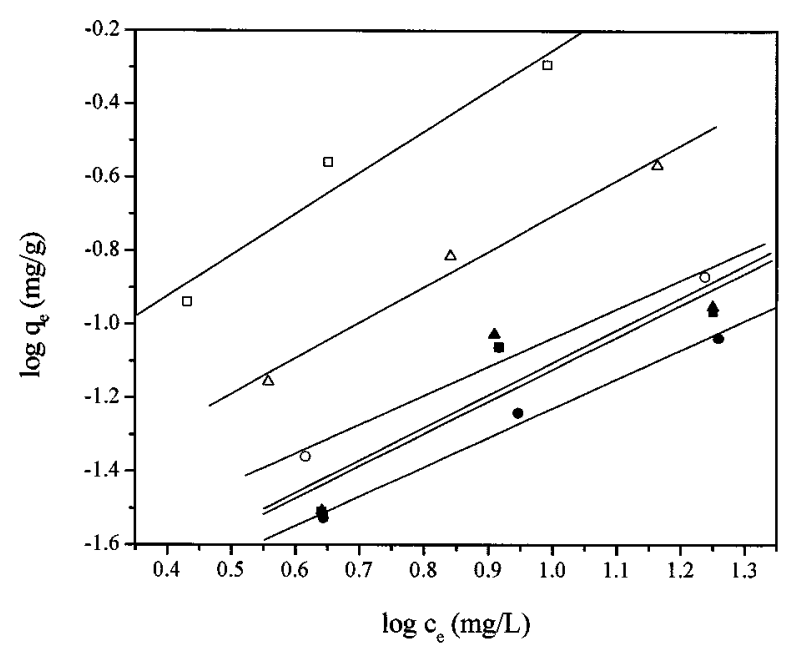

Fig. 4 Freundlich plots of individual adsorption isotherms for

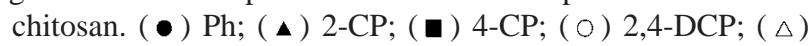
2,4,6-TCP; ( $\square$ ) PCP.

adsobent, $c_{\mathrm{e}}$ is the remaining concentration in the aqueous solution after adsorption, $K_{\mathrm{F}}$ is the Freundlich constant and $1 / n$ is an arbitrary parameter. A linearized form of this equation can be obtained as follows:

$$
\log q_{\mathrm{e}}=\log K_{\mathrm{F}}+(1 / n) \log c_{\mathrm{e}}
$$

A plot of $\log q_{\mathrm{e}} v s . \log c_{\mathrm{e}}$ would give $1 / n$ and $K_{\mathrm{F}}$ from the slope and intercept. The results are shown in Figs. 3 and 4 . The intercept, $K_{\mathrm{F}}$, is an indicator of adsorption ability and the slope, $1 / n$, is an indicator of concentration dependence of adsorption. The adsorption ability is higher when the value of $K_{\mathrm{F}}$ is larger.

Freundlich parameters and correlation coefficients obtained from Figs. 3 and 4 are listed in Table 1. As shown in Table 1 , the $K_{\mathrm{F}}$ values increases as the degree of chlorination increases for both sorbents. This tendency is the same as that of breakthrough volume measured. Comparing chitin and chitosan, the $K_{\mathrm{F}}$ values of chitosan are larger than those of chitin. This result may be due to the swelling of chitosan. Chitosan is more hydrophilic than chitin because of polar amine group; thus it swells in aqueous media and the active sites for hydrogen bonding are well developed. The similar slopes of the isotherm lines for each sorbent indicate

\begin{tabular}{|c|c|c|c|c|c|c|}
\hline & \multicolumn{3}{|c|}{ Chitin } & \multicolumn{3}{|c|}{ Chitosan } \\
\hline & $K_{\mathrm{F}}$ & $1 / n$ & $r$ & $K_{\mathrm{F}}$ & $1 / n$ & $r$ \\
\hline $\mathrm{Ph}$ & 0.00045 & 1.879 & 0.942 & 0.0094 & 0.797 & 0.995 \\
\hline 2-CP & 0.00062 & 1.976 & 0.984 & 0.0103 & 0.884 & 0.918 \\
\hline 4-CP & 0.00088 & 1.865 & 0.996 & 0.0100 & 0.874 & 0.894 \\
\hline 2,4-DCP & 0.00243 & 1.501 & 0.983 & 0.0151 & 0.785 & 0.989 \\
\hline 2,4,6-ТCP & 0.00203 & 1.594 & 0.992 & 0.0213 & 0.967 & 0.991 \\
\hline PCP & 0.01282 & 1.200 & 0.998 & 0.0426 & 1.118 & 0.974 \\
\hline
\end{tabular}

Table 1 Parameters and correlation coefficients for the Freundlich equation 
that the concentration dependence of phenols is similar without regard to the number of the substituents.

The present study has suggested the adsorption mechanism of phenols and chlorophenols for chitin and chitosan and the possibility of applying these sorbent materials to solid phase extraction. From the results of breakthrough volumes and adsorption isotherms, the main interaction in adsorption between the sorbents and phenols may be predicted to hydrogen bonding among electronegative atoms in chitin, chitosan or chlorophenols including chlorine, hydroxide or amine groups. Both chitin and chitosan may be useful in preconcentration of chlorophenols around environmental samples in the future.

This study was partially funded by grants at KIST through the Basic Science Research program (2E15471).

\section{References}

1. A. Martinou, D. Kafetzopoulos and V. Bouriotis, Carbohydr. Res., 273, 235 (1995).

2. Y. Shigemasa, H. Matsuura, H. Sashiwa and H. Saimoto,
Int. J. Biol. Macromol., 18, 237 (1996).

3. G. McKay, "Use of adsorbents for the removal of pollutants from wastewaters", CRC Press, Boca Raton, 1996.

4. H. Minamisawa, Y. Tatehaba, N. Arai and T. Okutani, Anal. Sci., 12, 947 (1996).

5. E. Onsoyen and O. Skaugrud, J. Chem. Technol. Biotechnol., 49, 395 (1990).

6. H. K. No and S. P. Meyers, J. Agric. Food Chem., 37, 580 (1989).

7. I. Safarik, Water Res., 29, 101 (1995).

8. M. G. Peter, J. Mol. Sci., Pure Appl. Chem., A32, 629 (1995).

9. S. Mima, M. Miya, R. Iwamoto and S. Yoshikawa, J. Appl. Polym. Sci., 28, 1909 (1983).

10. P. R. Austin, C. J. Brine, J. E. Castle and J. P. Zikakis, Science [Washington, D.C.], 212, 749 (1981).

11. R. L. Rouseff, G. R. Dettweiler, R. M. Swaine, M. Naim and U. Zehavi, J. Chromatogr. Sci., 30, 383 (1992).

12. M. Dressler, J. Chromatogr., 165, 167 (1979).

13. E. Matisova and S. Skrabakova, J. Chromatogr., 707, 145 (1995).

14. A. Di Corcia and R. Samperi, Anal. Chem., 62, 1490 (1990).

(Received March 30, 1998)

(Accepted August 6, 1998) 